\title{
Wolbachia Endosymbionts of Parasitic Nematodes
}

\author{
W.J. Kozek* and H. Figueroa-Marroquin**
}

*Department of Microbiology, Medical Sciences Campus, University of Puerto Rico, San Juan, Puerto Rico, 00936-5067

** Section of Oncocercosis, Guatemalan Ministry of Public Health and Welfare, Guatemala City, Guatemala

Bacterial endosymbionts, currently placed in the Wolbachia group of prokaryotes [1], have been discovered by the application of transmission electron microscopy to elucidate the ultrastructure of of filariae of man and animals[2-4]. These organisms are organotropic: they are found exclusively in the lateral chords (an osmoregulatory/secretory structure) of all larval stages and in adult male and female worms. In contrast to the Rickettsiae, which are found free in the cytoplasm, the Wolbachiae are found within cytopasmic vesicles enclosed by host membrane. They are pleomorphic, suggesting that they may have a complicated life cycle similar to the Chlamydiae [3], the bacterial forms are approximately $0.7 \mu \mathrm{m}$ in width by about $3 \mu \mathrm{m}$ in length, although smaller and larger forms are often seen, but these unusual sizes can be interpreted as different stages in the developmental cycle of Wolbachia. They are vertically transmitted from one generation of the parasite to another, are found in oocytes and, during intrauterine egg cleavage and embryonation of eggs into the microfilariae, they are present in the precursor cells of the hypodermis and of the female reproductive tract $[3,4]$.

Subsequent studies have demonstrated that 17 species of filariae, including those which are important parasites of man, harbor Wolbachia [5]. Demonstration of these organisms in pathogenic filariae required the change in our paradigm in the concept of filarial infections: it was necessary to consider filarial infections as a result of the relationship and interaction of three organisms Wolbachia-filaria-vertebrate host, rather than the classical filarial-vertebrate host relationship. Wolbachia appear to have a mutualistic relationship with their filarial hosts, and may contribute to inflammatory and some pathological effects observed during infection of humans with filariae $\{6,7]$. Since Wolbachia are susceptible to tetracylines, they can be considered as a possible target for chemotherapy and control of filarial infections [8].

The discovery of the presence of prokaryotic organisms in parasitic nematodes has opened a new area of investigation of host-parasite relationships. The eventual elucidation of this relationship will lead to better understanding of the pathological effects of filarial infections, and offer new approaches for control, treatment and eradiation of these diseases.

1. M. Sironi et al., Molec. Biochem. Parasitol. 74 (1995) 223.

2. D. McLaren at al., Trans. R. Soc. Trop. Med. Hyg. 69 (1975) 509.

3. W. J. Kozek and H. Figueroa Marroquin, Am. J. Trop. Med. Hyg. 26 (1977) 663

4. W. J. Kozek, J. Parasit. 63 (1977) 992.

5. R. Rao, Rec. Res. Devel. Exp. Med. 1 (2004) 95.

6. A. Saint Andre et al., Science 295 (2002) 1892.

7. M. J. Taylor. Ann. N.Y. Acad. Sci. 990 (2003) 444.

8. A. Hoerauf et al., Med. Microbiol. Immunol. 192 (2002) 211. 


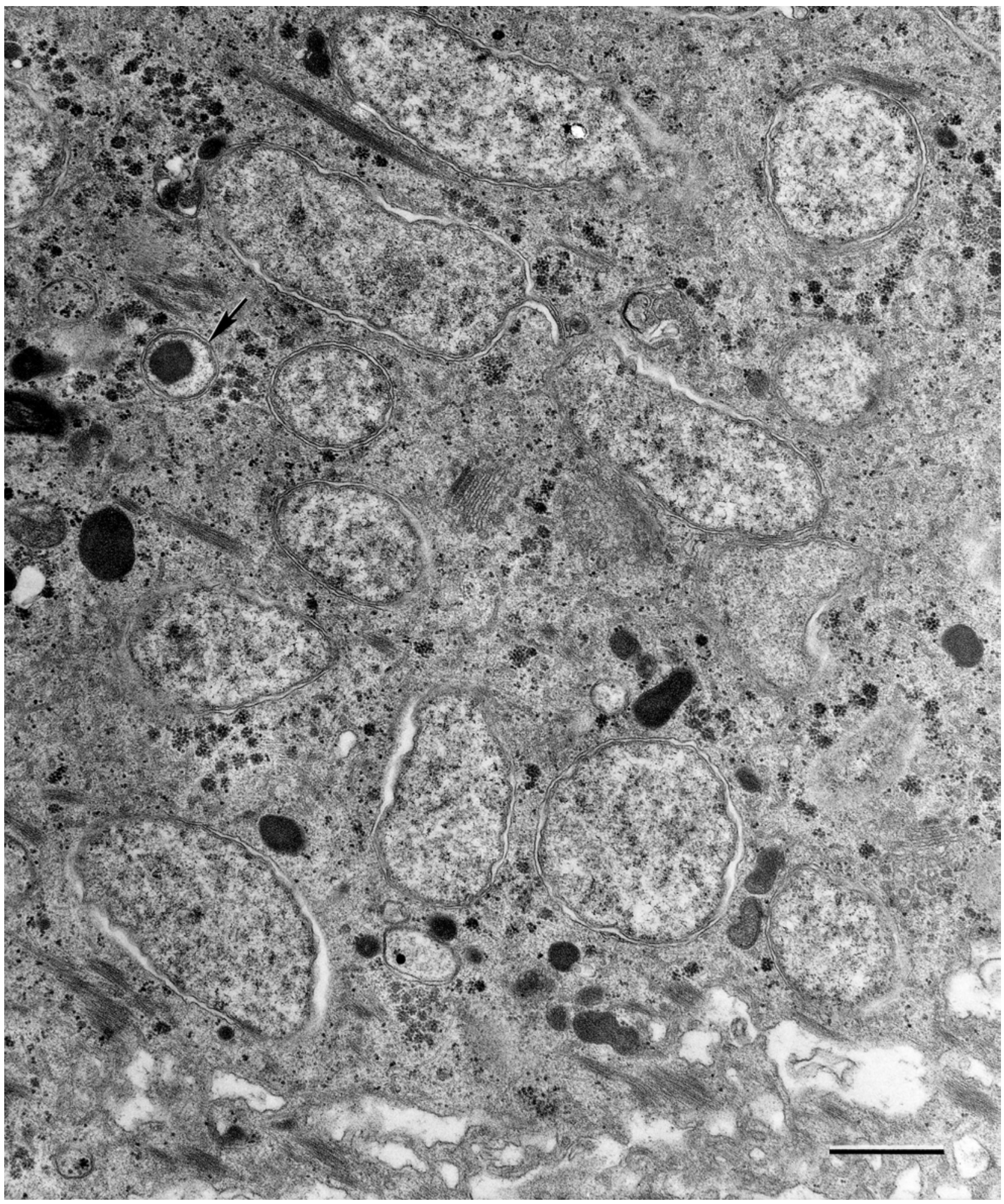

Fig. 1. Wolbachia in the lateral chord of Onchocerca volvulus. Arrow indicates a smaller form with dense inclusion, which may be analogous to the intermediate body of the Chlamydiae.

Scale bar $=1 \mu \mathrm{m}$. 